\title{
DANOS DE FRIO E QUALIDADE DE FRUTAS CÍTRICAS TRATADAS TERMICAMENTE E ARMAZENADAS SOB REFRIGERAÇÃ ${ }^{1}$
}

\author{
RICARDO ALFREDO KLUGE², MARIA LUIZA LYE JOMORI ${ }^{3}$, FERNANDO KAZUHIRO EDAGI ${ }^{4}$, \\ ANGELO PEDRO JACOMINO ${ }^{5}$, JUAN SAAVEDRA DEL AGUILA ${ }^{6}$
}

RESUMO -No presente trabalho, foram aplicados tratamentos térmicos (condicionamento térmico e aquecimento intermitente) em laranja 'Valência', tangor 'Murcott' e lima ácida 'Tahiti' armazenadas em baixa temperatura e avaliaram-se a incidência dos danos pelo frio e seus efeitos nas características físico-químicas das frutas. As frutas foram armazenadas durante 90 dias, a $1^{\circ} \mathrm{C}$, sendo avaliadas a cada 15 dias. A lima ácida 'Tahiti' e o tangor 'Murcott' suportaram até 90 dias de armazenamento, a $1^{\circ} \mathrm{C}$, com aquecimento intermitente, não apresentando danos pelo frio. No tratamento-controle (armazenamento contínuo a $1^{\circ} \mathrm{C}$ ), os danos pelo frio surgiram aos 30 dias de armazenamento para a lima 'Tahiti' e aos 45 dias para o tangor 'Murcott'. Em laranjas 'Valência', as injúrias pelo frio surgiram aos 45 dias de armazenamento, sendo significativamente menores no condicionamento térmico. O aquecimento intermitente é um tratamento que poder ser utilizado na conservação de frutas sem afetar suas características internas.

Termos para indexação: injúria pelo frio, Citrus sp., condicionamento térmico, aquecimento intermitente.

\section{CHILLING INJURY AND QUALITY OF CITRIC FRUITS SUBMITTED TO THERMAL TREATMENTS AND COLD STORAGE}

\begin{abstract}
In this study, thermal treatments (temperature conditioning and intermittent warming) were applied in 'Valencia' orange, 'Murcott' tangor and 'Tahiti' lime stored in low temperature. Fruits were stored at $1^{\circ} \mathrm{C}$ during 90 days and evaluate after every 15 days according with the incidence of chilling injury and changes in physical-chemical characteristics. 'Tahiti' lime and 'Murcott' tangor intermittently warmed could be stored up to 90 days at $1^{\circ} \mathrm{C}$, without the occurrence of chilling injury while control fruits showed chilling injury after 30 days ('Tahiti' lime) and after 45 days ('Murcott' tangor). 'Valencia' orange was affected by chilling injury after 45 days of cold storage. In this cultivar, temperature conditioning has caused lower incidence of chilling injury. The intermittent warming is a treatment that can be used in these citrus cultivars without affecting the physical and chemical characteristics of fruits.

Index terms: chilling injury, Citrus sp, temperature conditioning, intermittent warming.
\end{abstract}

\section{INTRODUÇÃO}

As frutas cítricas são bastante cultivadas no clima tropical de altitude, no interior do Estado de São Paulo, tendo como cultivares de destaque a laranja 'Valência', a lima ácida 'Tahiti' e o tangor 'Murcott'. O período de conservação refrigerado é variável para cada cultivar e depende da temperatura utilizada. Segundo dados de literatura, as condições ótimas para a conservação refrigerada de tangerinas são $4-7^{\circ} \mathrm{C}$ e $90-95 \%$ de umidade relativa (UR), com as frutas podendo ser armazenadas nestas condições por 2 a 4 semanas. Limas ácidas podem ser armazenadas a $9-10^{\circ} \mathrm{C}$ e $85-90 \%$ de UR, durante 6 a 8 semanas, enquanto para laranja, a temperatura recomendada é $3-9^{\circ} \mathrm{C}$ e $85-$ $90 \%$ de UR, com as frutas podendo ser armazenadas durante 3 a 8 semanas, dependendo da cultivar e das condições climáticas (Hardenburg et al., 1986; Chitarra \& Chitarra, 2005).

Atualmente, não existe nenhum método capaz de evitar completamente os danos de frio causados por armazenamento refrigerado. O método básico de controle consiste no armazenamento dos produtos em temperaturas adequadas, ou seja, acima da Temperatura Mínima de Segurança (TMS). Porém, esse procedimento pode não ser eficaz para longos períodos de armazenamento, situação em que os sintomas de injúrias pelo frio também podem manifestar-se (Kluge et al., 2001). Assim, outras alternativas vêm sendo testadas visando a diminuir ou a aliviar os danos de frio. As técnicas utilizadas reduzem os danos através do retardamento no desenvolvimento dos sintomas ou pelo aumento da tolerância do produto ao frio. Dentre essas técnicas, destacam-se os tratamentos térmicos, aplicados antes da refrigeração, na forma de condicionamento, ou durante o armazenamento refrigerado, na forma de aquecimento intermitente. $\mathrm{O}$ condicionamento térmico consiste em expor as frutas a temperaturas moderadas ou elevadas, por curtos períodos, antes de refrigerá-los. Esse tratamento tem reduzido os danos causados pelas baixas temperaturas e diminuído a

(Trabalho 055-06). Recebido em : 02-05-2006. Aceito para publicação em : 18-04-2007

${ }^{2}$ Departamento de Ciências Biológicas/ ESALQ-USP C. Postal 9, 13418-900, Piracicaba-SP. Bolsista do CNPq-Brasil. E-mail: rakluge@esalq.usp.br.

${ }^{3}$ Programa de Pós-Graduação em Fisiologia e Bioquímica de Plantas, ESALQ-USP, Piracicaba-SP. Bolsista da FAPESP. E-mail: ml_jomori@yahoo.com.br

${ }^{4}$ Curso de Engenharia Agronômica, ESALQ-USP, Piracicaba-SP. Bolsista da FAPESP. E-mail: fkedagi@esalq.usp.br

${ }^{5}$ Departamento de Produção Vegetal/ ESALQ-USP, Piracicaba-SP. Bolsista do CNPq. E-mail: jacomino@esalq.usp.br

${ }^{6}$ Programa de Pós-Graduação em Fitotecnia, ESALQ/USP, Piracicaba-SP. Bolsista da FAPESP. E-mail: jsaguila@esalq.usp.br 
incidência de podridões em frutas cítricas (Gonzalez-Aguilar et al., 1997 e 1998; Rodov et al., 1995 e 2000; Porat et al., 1999). Já o aquecimento intermitente consiste na interrupção da baixa temperatura de armazenamento, por um ou mais períodos com temperatura alta ou moderada. Esse tratamento deve ser realizado antes de os danos se tornarem irreversíveis, o que varia de acordo com o produto (Kluge et al., 2002). Foi verificada eficiência no controle de injúrias pelo frio com a aplicação do aquecimento intermitente em algumas frutas cítricas (Cohen, 1988; Schirra \& Mulas, 1995; Schirra \& Cohen, 1999).

A preocupação que se tem atualmente em relação aos tratamentos térmicos é seus efeitos sobre a qualidade interna dos frutos, sendo que o objetivo do presente trabalho foi avaliar o efeito dos tratamentos térmicos sobre a conservação das frutas de três cultivares de citros mantidas sob temperatura baixa $\left(1^{\circ} \mathrm{C}\right)$, analisando-se a incidência de injúrias pelo frio e suas principais características físico-químicas.

\section{MATERIAL E MÉTODOS}

Frutos de lima ácida 'Tahiti', laranja 'Valência' e tangor 'Murcott', colhidos na região de Mogi Mirim-SP, foram cuidadosamente transportados até o Laboratório de Fisiologia e Bioquímica Pós-colheita do Departamento de Ciências Biológicas, da Escola Superior de Agricultura "Luiz de Queiroz" - ESALQ/ USP, em Piracicaba-SP, Brasil, onde foram selecionados quanto à firmeza, ausência de danos mecânicos e infecções visíveis.

Os seguintes tratamentos foram aplicados: T1- Frutas sem tratamento e armazenadas a $1^{\circ} \mathrm{C}$ (Controle); T2- Aquecimento rápido das frutas em água quente a $53^{\circ} \mathrm{C}$, durante 3 minutos e armazenamento a $1^{\circ} \mathrm{C}$; T3- Aquecimento lento das frutas, com colocação das mesmas em câmara a $37^{\circ} \mathrm{C}$, por 2 dias, antes de serem armazenadas a $1^{\circ} \mathrm{C}$, e T4- Aquecimento intermitente, com ciclos de 6 dias a $1^{\circ} \mathrm{C}$ e 1 dia a $25^{\circ} \mathrm{C}$.

As laranjas e tangores foram armazenadas a $1^{\circ} \mathrm{C}$, por 15 ; $30 ; 45 ; 60,75$ e 90 dias, enquanto as limas ácidas 'Tahiti' foram armazenadas até o $45^{\circ}$ dia. Após cada período de armazenamento, elas eram expostas à temperatura ambiente $\left(25^{\circ} \mathrm{C}\right)$ por 3 dias, para simular uma comercialização.

O delineamento experimental foi inteiramente ao acaso, em esquema fatorial $8 \times 6$. Os fatores estudados foram tratamentos em oito níveis, e o período de armazenamento, em seis níveis. Foram utilizadas quatro repetições de 10 frutas.

Foram determinadas as injúrias pelo frio, pela determinação da porcentagem de frutas injuriadas pelo frio (depressões na casca) e estimado o índice de injúrias (severidade), de acordo com a metodologia adaptada de Sala \& LaFuente (1999). Relativamente à superfície da casca afetada, as frutas foram classificadas em cinco categorias: $0=$ sem depressões superficiais $(0 \%$ da superfície afetada); $1=$ pouco $(1$ a $5 \%$ da superfície afetada $) ; 2=$ médio $(5-25 \%) ; 3=\operatorname{severo}(25-50 \%)$, e $4=$ muito severo $(>50 \%$ da superfície afetada).

As análises de qualidade avaliadas, realizadas conforme o indicado por Carvalho et al. (1990), foram: Porcentagem de suco, em que cada repetição foi pesada e teve seu suco extraído, permitindo que a porcentagem de suco fosse calculada através da fórmula: $\%$ de suco $=(\mathrm{MS} / \mathrm{MF})$ x 100 , onde $\mathrm{MS}=$ massa do suco $(\mathrm{g})$ e $\mathrm{MF}=$ massa da fruta; Teor de sólidos solúveis (SS), determinado em refratômetro digital, com correção automática de temperatura para $20^{\circ} \mathrm{C}$ e os resultados expressos em ${ }^{\circ} \mathrm{Brix}$; Acidez titulável (AT), com a utilização de $10 \mathrm{~mL}$ do suco diluídos com $90 \mathrm{~mL}$ de água destilada, que foi titulado potenciometricamente com $\mathrm{NaOH}$ a 0,1 n até $\mathrm{pH} 8,10$ e os resultados expressos em $\%$ de ácido cítrico; "Ratio", calculado pela relação entre o teor de SS e o de AT; Ácido ascórbico (vitamina C), em que uma alíquota de $10 \mathrm{~mL}$ de suco foi diluída com $50 \mathrm{~mL}$ de ácido oxálico, e em seguida titulado com o 2,6-diclorofenol indolfenol-sódico (DCFI) até a coloração rosada persistente por 15 segundos, com os resultados expressos em mg de ácido ascórbico por $100 \mathrm{~mL}$ de suco.

Os resultados coletados foram submetidos à análise de variância (teste F), e a determinação da diferença mínima significativa (d.m.s.) foi calculada. As diferenças entre dois tratamentos maiores que a soma de dois desvios-padrão foram consideradas significativas a $5 \%$ de probabilidade.

\section{RESULTADOS E DISCUSSÃO}

Em laranjas 'Valência', as injúrias pelo frio surgiram aos 45 dias de armazenamento, sendo significativamente menores nas condicionadas termicamente. Para a lima 'Tahiti' e o tangor 'Murcott', os danos pelo frio surgiram aos 30 e 45 dias, respectivamente. $\mathrm{O}$ condicionamento térmico lento $\left(37^{\circ} \mathrm{C}\right.$ durante 2 dias) ocasionou queimaduras na casca da laranja 'Valência' (em mais de $80 \%$ das frutas), não associadas ao frio, mas ao estresse resultante da alta temperatura, à qual as frutas foram submetidas antes da refrigeração. Este tipo de dano foi confirmado em testes subseqüentes e não ocorreu na lima 'Tahiti' e no tangor 'Murcott'.

$\mathrm{O}$ aquecimento intermitente, de maneira geral, proporcionou as menores incidências de injúrias pelo frio, na lima 'Tahiti' e no tangor 'Murcott', sendo que, nesta última, o aquecimento lento foi igualmente eficaz (Figura 1). A redução de injúrias pelo frio, através de tratamentos térmicos, também foi observada por outros autores (Gonzalez-Aguilar et al., 1997 e 1998; Rodov et al., 1995 e 2000; Porat et al., 1999).

A lima 'Tahiti' suportou até 90 dias de armazenamento quando aplicado o tratamento com aquecimento intermitente, enquanto para os outros tratamentos as frutas foram sendo descartadas, devido à alta incidência de injúrias pelo frio.

De maneira geral, observou-se que, ao longo do armazenamento, a porcentagem de suco diminuiu, porém, nos frutos de Laranja 'Valência', observou-se um ligeiro acréscimo ao final do experimento (Tabela 1). Aos 30 dias de armazenamento, observa-se um aumento na porcentagem de suco nos frutos cítricos submetidos aos tratamentos térmicos. Esse fato pode ter sido devido à perda de água por transpiração, que aumenta a relação de suco sobre a massa total dos frutos, pois a transpiração fica restrita apenas no flavedo, não interferindo na quantidade de suco (Jomori et al., 2003).

O teor de sólidos solúveis teve pequena variação durante o experimento, de 7,82 a $8,82^{\circ}$ Brix em lima 'Tahiti', de 10,40 a $11,75^{\circ}$ Brix em tangor 'Murcott' e de 11,47 a $12,77^{\circ}$ Brix na laranja 
'Valência' (Tabela 2). Observou-se que, ao longo do armazenamento, houve decréscimo do teor de sólidos solúveis. Esse teor é um indicativo da quantidade de açúcares existentes na fruta, além de compostos como ácidos, vitaminas, aminoácidos e algumas pectinas (Kluge et al., 2002).

De modo geral, houve decréscimo nos teores de acidez titulável ao longo do armazenamento (Tabela 3). Os frutos submetidos ao condicionamento térmico, tanto rápido como lento, obtiveram menor teor de acidez, resultado semelhante observado em maçãs por Liu (1978).

O "Ratio" apresentou pequena variação durante o experimento, tendo os valores oscilados entre 1,37 e 1,64 em lima 'Tahiti', entre 10,79 e 14,87 em tangor 'Murcott' e entre 9,24 e 11,13 na laranja 'Valência' (Tabela 4). Ao longo do armazenamento, houve um pequeno aumento do "ratio", devido à relação existente entre o teor de SS e a AT, onde a acidez se reduziu mais rapidamente que o teor de $\mathrm{SS}$.

O teor de ácido ascórbico é um importante atributo de qualidade dos frutos cítricos em geral e, de modo geral, observouse redução no seu teor durante o armazenamento de até $20 \%$ em lima 'Tahiti', de até $62 \%$ em tangor 'Murcott' e de até $38 \%$ na laranja 'Valência' (Tabela 5). Essa redução pode estar relacionada com a própria senescência do fruto, onde o ácido ascórbico é consumido em reações oxidativas (Gardner et al., 2000).

O aquecimento lento consumiu parte do teor de ácido ascórbico na laranja 'Valência' e no tangor 'Murcott', o que pode trazer prejuízos à qualidade nutricional da fruta, em termos de vitamina $\mathrm{C}$ (Tabela 5). O aquecimento intermitente foi o tratamento que proporcionou menores reduções no teor de ácido ascórbico, provavelmente devido às menores reações antioxidativas resultante da menor manifestação de injúrias pelo frio.
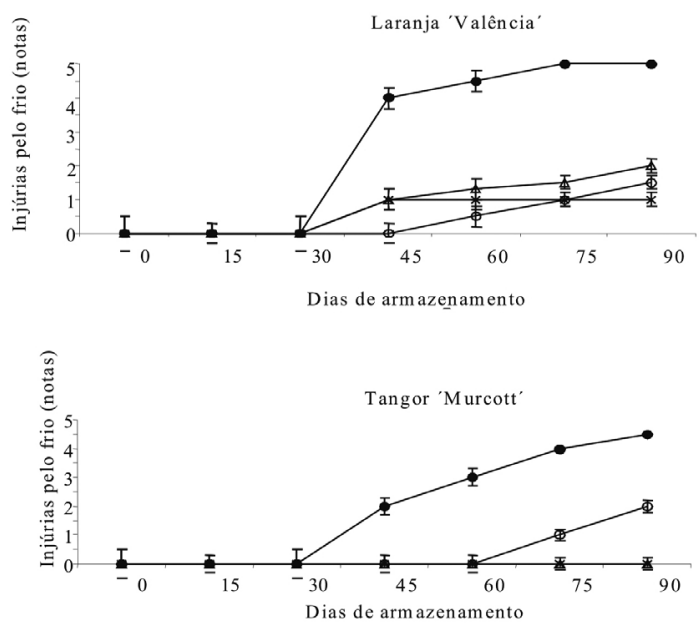

$-$

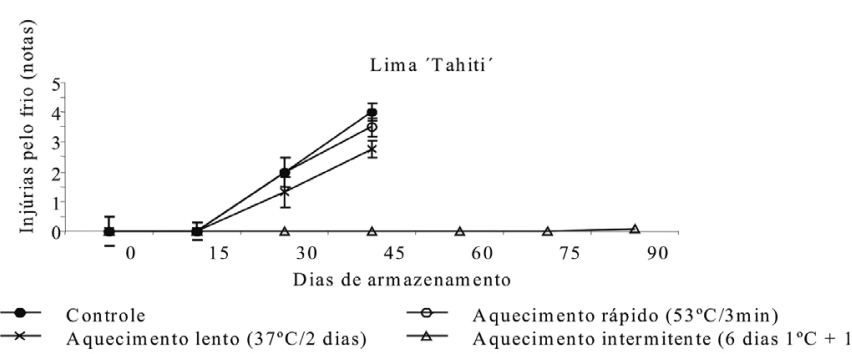

FIGURA 1 - Efeito dos diferentes tratamentos sobre as injúrias pelo frio (notas), em laranja 'Valência', tangor 'Murcott' e lima ácida 'Tahiti'. Notas: $0=$ sem depressões superficiais ( $0 \%$ da superfície afetada); $1=$ pouco ( 1 a $5 \%$ da superfície afetada), $2=$ médio $(5-25 \%) ; 3=$ severo $(25-50 \%)$, e $4=$ muito severo ( $>50 \%$ da superfície afetada). Barras verticais representam o desvio padrão da média $(n=4)$.

TABELA 1 - Porcentagem de suco em laranja 'Valência', tangor 'Murcott' e lima ácida 'Tahiti' armazenadas a $1^{\circ} \mathrm{C}$ após terem sido submetidos a diferentes tratamentos ${ }^{1}$.

\begin{tabular}{|c|c|c|c|c|c|c|c|}
\hline \multirow[b]{2}{*}{ Tratamentos } & \multicolumn{7}{|c|}{ Tempo de armazenamento a $1^{\circ} \mathrm{C}$} \\
\hline & Inicial & 15 dias & 30 dias & 45 dias & 60 dias & 75 dias & 90 dias \\
\hline & \multicolumn{7}{|c|}{ Laranja'Valência' } \\
\hline Controle & 52,98 & 56,79 & 50,00 & 55,84 & 55,78 & 52,13 & 54,65 \\
\hline Aquecimento rápido $\left(53^{\circ} \mathrm{C} / 3 \mathrm{~min}\right)$ & 52,98 & 53,42 & 56,96 & 51,98 & 55,11 & 53,17 & 56,04 \\
\hline Aquecimento lento $\left(37^{\circ} \mathrm{C} / 2\right.$ dias $)$ & 52,98 & 51,96 & 54,01 & 52,07 & 53,93 & 51,34 & 50,52 \\
\hline Aquecimento intermitente & & & & & & & \\
\hline \multirow[t]{2}{*}{$\left(6\right.$ dias $1^{\circ} \mathrm{C}+1$ dia $\left.25^{\circ} \mathrm{C}\right)$} & 52,98 & 51,40 & 56,29 & 52,84 & 53,58 & 53,10 & 52,17 \\
\hline & \multicolumn{7}{|c|}{ Tangor 'Murcott' } \\
\hline Controle & 53,03 & 54,81 & 52,87 & 49,08 & 50,01 & 49.89 & 48,95 \\
\hline Aquecimento rápido $\left(53^{\circ} \mathrm{C} / 3 \mathrm{~min}\right)$ & 53,03 & 53,05 & 54,80 & 53,15 & 50,45 & 51,40 & 48,00 \\
\hline Aquecimento lento $\left(37^{\circ} \mathrm{C} / 2\right.$ dias $)$ & 53,03 & 54,00 & 53,45 & 54,80 & 49,09 & 50,87 & 48,76 \\
\hline Aquecimento intermitente & & & & & & & \\
\hline \multirow[t]{2}{*}{$\left(6\right.$ dias $1^{\circ} \mathrm{C}+1$ dia $\left.25^{\circ} \mathrm{C}\right)$} & 53,03 & 53,85 & 50,42 & 51,44 & 49.08 & 50,01 & 50,08 \\
\hline & \multicolumn{7}{|c|}{ Lima 'Tahiti' } \\
\hline Controle & 47,43 & 44,14 & 42,66 & 36,01 & - & - & - \\
\hline Aquecimento rápido $\left(53^{\circ} \mathrm{C} / 3 \mathrm{~min}\right)$ & 47,43 & 43,75 & 45,48 & 36,83 & - & - & - \\
\hline Aquecimento lento $\left(37^{\circ} \mathrm{C} / 2\right.$ dias $)$ & 47,43 & 42,08 & 47,04 & 39,30 & - & - & - \\
\hline $\begin{array}{l}\text { Aquecimento intermitente } \\
\left(6 \text { dias } 1^{\circ} \mathrm{C}+1 \text { dia } 25^{\circ} \mathrm{C}\right)\end{array}$ & 47,43 & 45,44 & 48,93 & 48,38 & - & - & - \\
\hline
\end{tabular}

${ }^{1}$ Teste $\mathrm{F}$ não significativo para efeitos isolados de tratamentos e tempo de armazenamento e interação entre os dois fatores. C.V. $=6,78 \%$ (laranja 'Valência'); 10,90\% (tangor 'Murcott'); 12,34\% (lima 'Tahiti'). 
TABELA 2 - Teor de sólidos solúveis (SS) de laranja 'Valência', tangor 'Murcott' e lima ácida 'Tahiti' armazenadas a $1{ }^{\circ} \mathrm{C}$ após terem sido submetidos a diferentes tratamentos'.

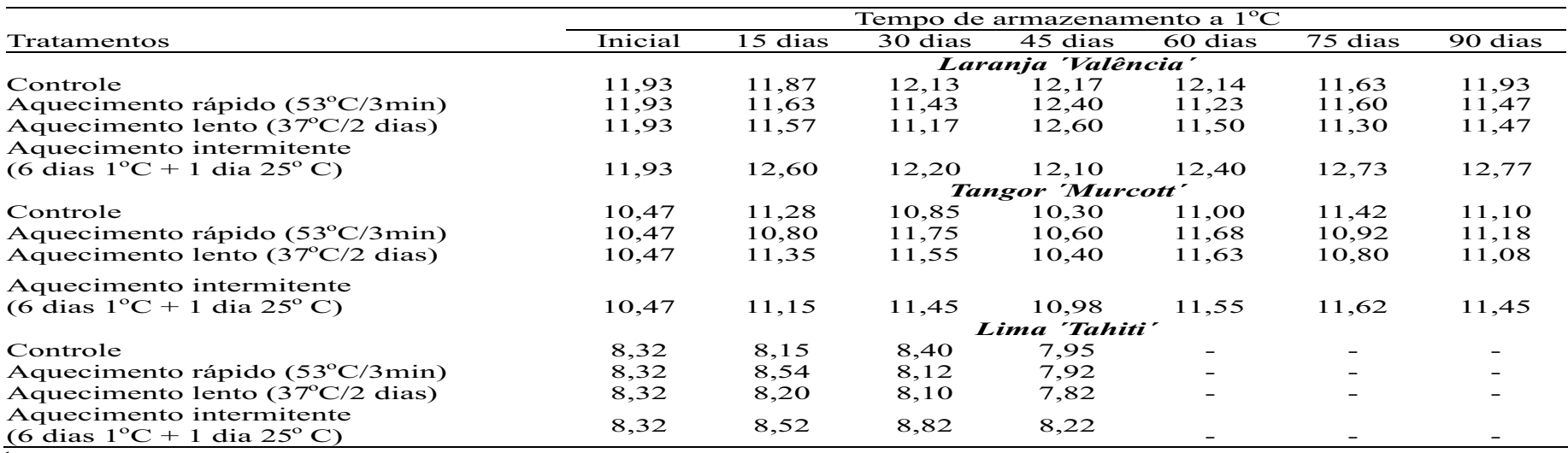

${ }^{1}$ Teste $\mathrm{F}$ não significativo para efeitos isolados de tratamentos e tempo de armazenamento e interação entre os dois fatores. C.V. = 3,45\% (laranja

'Valência'); 4,93\% (tangor 'Murcott'); 5,21\% (lima 'Tahiti').

TABELA 3 - Acidez titulável (\% de ácido cítrico) de laranja 'Valência', tangor 'Murcott' e lima ácida 'Tahiti' armazenadas a $1^{\circ} \mathrm{C}$ após terem sido submetidos a diferentes tratamentos'.

\begin{tabular}{|c|c|c|c|c|c|c|c|}
\hline \multirow[b]{2}{*}{ Tratamentos } & \multicolumn{7}{|c|}{ Tempo de armazenamento a $1^{\circ} \mathrm{C}$} \\
\hline & Inicial & 15 dias & 30 dias & 45 dias & 60 dias & 75 dias & 90 dias \\
\hline & \multicolumn{7}{|c|}{ Laranja'Valência' } \\
\hline Controle & 1,23 & 1,30 & 1,26 & 1,27 & 1,27 & 1,18 & 1,30 \\
\hline Aquecimento rápido $\left(53^{\circ} \mathrm{C} / 3 \mathrm{~min}\right)$ & 1,23 & 1,18 & 1,11 & 1,17 & 1,14 & 1,04 & 1,14 \\
\hline Aquecimento lento $\left(37^{\circ} \mathrm{C} / 2\right.$ dias $)$ & 1,23 & 1,22 & 1,12 & 1,13 & 1,18 & 1,15 & 1,14 \\
\hline \multirow{3}{*}{$\left(6\right.$ dias $1^{\circ} \mathrm{C}+1$ dia $\left.25^{\circ} \mathrm{C}\right)$} & & & & & & & \\
\hline & 1,23 & 1,35 & 1,31 & 1,16 & 1,27 & 1,24 & 1,19 \\
\hline & \multicolumn{7}{|c|}{ Tangor 'Murcott' } \\
\hline Controle & 0,97 & 0,95 & 0,88 & 0,92 & 0,89 & 0,89 & 0,77 \\
\hline Aquecimento rápido $\left(53^{\circ} \mathrm{C} / 3 \mathrm{~min}\right)$ & 0,97 & 0,92 & 0,91 & 0,85 & 0,83 & 0,82 & 0,76 \\
\hline Aquecimento lento $\left(37^{\circ} \mathrm{C} / 2\right.$ dias $)$ & 0,97 & 0,87 & 0,77 & 0,79 & 0,80 & 0,79 & 0,78 \\
\hline \multirow[t]{2}{*}{$\left(6\right.$ dias $1^{\circ} \mathrm{C}+1$ dia $\left.25^{\circ} \mathrm{C}\right)$} & 0,97 & 0,97 & 0,80 & 0,82 & 0,80 & 0,80 & 0,77 \\
\hline & \multicolumn{7}{|c|}{ Lima 'Tahiti' } \\
\hline Controle & 6,00 & 6,20 & 5,61 & 5,27 & - & - & - \\
\hline Aquecimento rápido $\left(53^{\circ} \mathrm{C} / 3 \mathrm{~min}\right)$ & 6,00 & 5,67 & 5,93 & 4,84 & - & - & - \\
\hline Aquecimento lento $\left(37^{\circ} \mathrm{C} / 2\right.$ dias $)$ & 6,00 & 5,65 & 5,63 & 5,17 & - & - & - \\
\hline $\begin{array}{l}\text { Aquecimento intermitente } \\
\left(6 \text { dias } 1^{\circ} \mathrm{C}+1 \text { dia } 25^{\circ} \mathrm{C}\right)\end{array}$ & 6,00 & 5,92 & 5,66 & 6,07 & - & - & - \\
\hline
\end{tabular}

${ }^{1}$ Teste $\mathrm{F}$ não significativo para efeitos isolados de tratamentos e tempo de armazenamento e interação entre os dois fatores. C.V. $=6,11 \%$ (laranja

'Valência'); 4,44\% (tangor 'Murcott'); 3,17\% (lima 'Tahiti').

TABELA 4 - "Ratio”' (SS/AT) de laranja 'Valência', tangor 'Murcott' e lima ácida 'Tahiti’ armazenadas a $1^{\circ} \mathrm{C}$ após terem sido submetidos a diferentes tratamentos ${ }^{1}$.

\begin{tabular}{|c|c|c|c|c|c|c|c|}
\hline \multirow[b]{2}{*}{ Tratamentos } & \multicolumn{7}{|c|}{ Tempo de armazenamento a $1^{\circ} \mathrm{C}$} \\
\hline & Inicial & 15 dias & 30 dias & 45 dias & 60 dias & 75 dias & 90 dias \\
\hline & \multicolumn{7}{|c|}{ Laranja'Valência' } \\
\hline Controle & 9,84 & 9,24 & 9,66 & 9,59 & 9,62 & 9,93 & 9,21 \\
\hline Aquecimento rápido $\left(53^{\circ} \mathrm{C} / 3 \mathrm{~min}\right)$ & 9,84 & 9,87 & 10,38 & 10,62 & 9,92 & 11,17 & 10,08 \\
\hline Aquecimento lento $\left(37^{\circ} \mathrm{C} / 2\right.$ dias $)$ & 9,84 & 9,46 & 9,99 & 11,13 & 9,82 & 9,88 & 10,06 \\
\hline Aquecimento intermitente & & & & & & & \\
\hline \multirow{2}{*}{$\begin{array}{l}\text { Aquecimento intermitente } \\
\left(6 \text { dias } 1^{\circ} \mathrm{C}+1 \text { dia } 25^{\circ} \mathrm{C}\right)\end{array}$} & 9,84 & 9,36 & 9,36 & 10,41 & 9,82 & 10,32 & 10,73 \\
\hline & \multicolumn{7}{|c|}{ Tangor 'Murcott' } \\
\hline Controle & 10,79 & 11,87 & 12,33 & 11,20 & 12,36 & 12,83 & 14,42 \\
\hline Aquecimento rápido $\left(53^{\circ} \mathrm{C} / 3 \mathrm{~min}\right)$ & 10,79 & 11,74 & 12,91 & 12,47 & 14,07 & 13,32 & 14,71 \\
\hline Aquecimento lento $\left(37^{\circ} \mathrm{C} / 2\right.$ dias $)$ & 10,79 & 13,05 & 15,00 & 13,16 & 14,54 & 13,67 & 14,21 \\
\hline Aquecimento intermitente & & & & & & & \\
\hline \multirow{2}{*}{$\left(6\right.$ dias $1^{\circ} \mathrm{C}+1$ dia $\left.25^{\circ} \mathrm{C}\right)$} & 10,79 & 11,49 & 14,31 & 13,39 & 14,44 & 14,53 & 14,87 \\
\hline & \multicolumn{7}{|c|}{ Lima 'Tahiti' } \\
\hline Controle & 1,39 & 1,31 & 1,50 & 1,51 & - & - & - \\
\hline Aquecimento rápido $\left(53^{\circ} \mathrm{C} / 3 \mathrm{~min}\right)$ & 1,39 & 1,51 & 1,37 & 1,64 & - & - & - \\
\hline Aquecimento lento $\left(37^{\circ} \mathrm{C} / 2\right.$ dias $)$ & 1,39 & 1,45 & 1,44 & 1,51 & - & - & - \\
\hline $\begin{array}{l}\text { Aquecimento intermitente } \\
\left(6 \text { dias } 1^{\circ} \mathrm{C}+1 \text { dia } 25^{\circ} \mathrm{C}\right)\end{array}$ & 1,39 & 1,44 & 1,56 & 1,35 & - & - & - \\
\hline
\end{tabular}

${ }^{1}$ Teste F não significativo para efeitos isolados de tratamentos e tempo de armazenamento e interação entre os dois fatores. C.V. = 3,66\% (laranja 'Valência'); 4,19\% (tangor 'Murcott'); 3,04\% (lima 'Tahiti'). 
TABELA 5 - Teor de ácido ascórbico $\left(\mathrm{mg} 100 \mathrm{~g}^{-1}\right)$ de laranja 'Valência', tangor 'Murcott' e lima ácida 'Tahiti' armazenadas a $1^{\circ} \mathrm{C}$ após terem sido submetidos a diferentes tratamentos'.

\begin{tabular}{|c|c|c|c|c|c|c|c|}
\hline \multirow[b]{2}{*}{ Tratamentos } & \multicolumn{7}{|c|}{ Tempo de armazenamento a $1^{\circ} \mathrm{C}$} \\
\hline & Inicial & 15 dias & 30 dias & 45 dias & 60 dias & 75 dias & 90 dias \\
\hline & \multicolumn{7}{|c|}{ Laranja 'Valência' } \\
\hline Controle & $63,20 \mathrm{aA}$ & 59,09 aA & $56,00 \mathrm{aAB}$ & $55,89 \mathrm{aAB}$ & $54,00 \mathrm{aB}$ & $50,23 \mathrm{aBC}$ & $45,67 \mathrm{bcC}$ \\
\hline Aquecimento rápido $\left(53^{\circ} \mathrm{C} / 3 \mathrm{~min}\right)$ & $63,20 \mathrm{aA}$ & $58,00 \mathrm{aABC}$ & $59,07 \mathrm{aABC}$ & $54,98 \mathrm{aB}$ & $53,22 \mathrm{aB}$ & $53,67 \mathrm{aB}$ & $51,22 \mathrm{abB}$ \\
\hline Aquecimento lento $\left(37^{\circ} \mathrm{C} / 2\right.$ dias $)$ & $63,20 \mathrm{aA}$ & $44,00 \mathrm{bB}$ & $41,34 \mathrm{bB}$ & $42,36 \mathrm{bB}$ & $43,55 \mathrm{bB}$ & $40,65 \mathrm{~B}$ & $39,34 \mathrm{cB}$ \\
\hline \multirow[t]{2}{*}{$\left(6 \operatorname{dias} 1^{\circ} \mathrm{C}+1\right.$ dia $\left.25^{\circ} \mathrm{C}\right)$} & $63,20 \mathrm{aA}$ & $60,01 \mathrm{aA}$ & 59,89 aA & 59,88 aA & $57,65 \mathrm{aA}$ & $56,34 \mathrm{aA}$ & $57,99 \mathrm{abA}$ \\
\hline & \multicolumn{7}{|c|}{ Tangor 'Murcott' } \\
\hline Controle & $40,05 \mathrm{aA}$ & 38,90 aA & 36,09 aA & $37,11 \mathrm{aA}$ & $36,65 \mathrm{aA}$ & 34,99 aA & 33,12 aA \\
\hline Aquecimento rápido $\left(53^{\circ} \mathrm{C} / 3 \mathrm{~min}\right)$ & $40,05 \mathrm{aA}$ & 34,67 aA & 34,56 aA & 33,88 aA & 34,56 aA & 32,55 aA & $32,00 \mathrm{aA}$ \\
\hline Aquecimento lento $\left(37^{\circ} \mathrm{C} / 2\right.$ dias $)$ & $40,05 \mathrm{aA}$ & $20,06 \mathrm{bB}$ & $19,67 \mathrm{bB}$ & $18,96 \mathrm{bB}$ & $18,90 \mathrm{bB}$ & $17,09 \mathrm{bB}$ & $15,09 \mathrm{bB}$ \\
\hline $\begin{array}{l}\text { Aquecimento intermitente } \\
\left(6 \text { dias } 1^{\circ} \mathrm{C}+1 \text { dia } 25^{\circ} \mathrm{C}\right)\end{array}$ & 40,05 aA & 39,09 aA & 38,77 aA & $39,00 \mathrm{aA}$ & 37,88 aA & $37,00 \mathrm{aA}$ & 38,93 aA \\
\hline & \multicolumn{7}{|c|}{ Lima 'Tahiti' } \\
\hline Controle & $6,00 \mathrm{aA}$ & $6,20 \mathrm{aA}$ & 5,61 aA & 5,27 aA & - & - & - \\
\hline Aquecimento rápido $\left(53^{\circ} \mathrm{C} / 3 \mathrm{~min}\right)$ & $6,00 \mathrm{aA}$ & $5,67 \mathrm{aA}$ & $5,93 \mathrm{aA}$ & $5,84 \mathrm{aA}$ & - & - & - \\
\hline Aquecimento lento $\left(37^{\circ} \mathrm{C} / 2\right.$ dias $)$ & $6,00 \mathrm{aA}$ & $5,65 \mathrm{aA}$ & $5,63 \mathrm{aA}$ & 5,17 aA & - & - & - \\
\hline $\begin{array}{l}\text { Aquecimento intermitente } \\
\left(6 \text { dias } 1^{\circ} \mathrm{C}+1 \text { dia } 25^{\circ} \mathrm{C}\right)\end{array}$ & $6,00 \mathrm{aA}$ & $5,92 \mathrm{aA}$ & $5,66 \mathrm{aA}$ & $6,07 \mathrm{aA}$ & - & - & - \\
\hline
\end{tabular}

${ }^{1}$ Para cada cultivar, médias seguidas de mesma letra minúscula na coluna e maiúscula na linha não diferem entre si pelo teste de Tukey a $5 \%$ de probabilidade. C.V. = 9,61\% (laranja 'Valência'); 10,33\% (tangor 'Murcott'); 4,22\% (lima 'Tahiti').

\section{CONCLUSÃO}

$\mathrm{O}$ aquecimento intermitente, utilizado como tratamento no armazenamento sob refrigeração de frutas cítricas, a $1{ }^{\circ} \mathrm{C}$ e 90 $95 \%$ UR, reduz as injúrias pelo frio e pode prolongar a conservação da laranja "Valência", sendo também eficiente para o tangor "Murcott" e a lima "Tahiti", sem afetar as características internas destes.

\section{AGRADECIMENTOS}

Os autores agradecem à Fundação de Amparo à Pesquisa do Estado de São Paulo (FAPESP), pelo suporte financeiro fornecido a este trabalho (proc. $\mathrm{N}^{\circ} 01 / 13433-5$ ) e ao Conselho Nacional de Desenvolvimento Científico e Tecnológico (CNPq), pela Bolsa de Produtividade em Pesquisa e Auxílio à Pesquisa (Projeto Universal) ao primeiro autor.

\section{REFERÊNCIAS}

CARVALHO, C.R.L.; MANTOVANI, D.M.B.; CARVALHO, P.R.N.; MORAES, R.M.N. Análises químicas de alimentos. Campinas: ITAL, 1990. 121p. (Manual Técnico)

COHEN, E. Commercial use of long-term storage of lemon with intermittent warming. HortScience, Alexandria, v.23, n.2, p. 400, 1988.

CHITARRA, M.I. ; CHITARRA, A.B. Pós-colheita de frutos e hortaliças: fisiologia e manuseio. 2. ed. Lavras: ESAL/FAEPE, 2005. 783p.

GARDNER, P.T.; WHITE, T.A.C.; McPHAIL, D.B.; DUTHIE, G.G. The relative contribuitions of vitamin $\mathrm{C}$, carotenoids and phenolic to the antioxidants potencial of fruits juices. Food Chemistry, Barking, v.68, p.471-474, 2000.

GONZALEZ-AGUILAR, G.A.; ZACARIAS, L.; LAFUENTE, M.T. Ripening affects high-temperature-induced polyamines and their changes during cold storage of hybrid Fortune mandarins. Journal of Agriculture and Food Chemistry, Easton, v.46, p.3503-3508, 1998.

GONZALEZ-AGUILAR, G.A.; ZACARIAS, L.; MULAS, M.; LAFUENTE, M.T. Temperature and duration of water dips influence chilling injury, decay and polyamine content in 'Fortune' mandarins. Portharvest Biology and Technology, Wageningen, v.12, p.61-69, 1997.

HARDENBURG, R.E.; WATADA, A.E.; WANG, C.Y. The commercial storage of fruits, vegetables, and florist, and nursery stocks. Washington: USDA, 1986. 130p. (Agriculture Handbook, 66)

JOMORI, M.L.L.; KLUGE, R.A.; JACOMINO, A.P.; TAVARES, S. Conservação refrigerada de lima ácida "Tahití": uso de 1metilciclorpopeno, ácido giberélico e cera. Revista Brasileira de Fruticultura, Jaboticabal, v.25, n.3, p.406-409, 2003.

KLUGE, R.A.; NACHTIGAL, J.C.; FACHINELLO, J.C.; BILHALVA, A.B. Fisiologia e manejo pós-colheita de frutas de clima temperado. 2. ed. Campinas: Livraria e Editora Rural, 2002.214p.

KLUGE, R.A.; SCARPARE FILHO, J.A.; JACOMINO, A.P.; PEIXOTO, C.P. Distúrbios fisiológicos em frutos. Piracicaba :FEALQ, 2001. 58p.

LIU, F.W. Modification of apple quality by high-temperature. Journal of the American Society for Horticultural Science, Alexandria, v.103, n.3, p.730-732, 1978.

PORAT, R.; WEISS, B.; COHEN, L.; DAUS, A.; DROBY, S. Effects 
of ethylene and 1-methylcyclopropene on the postharvest qualities of 'Shamouti' oranges. Postharvest Biology and Technology, Wageningen, v.15, n.2, p.155-163, 1999.

RODOV, V.; AGAR, T.; PERETZ, J.; NAFUSSI, B.; KIM, J.J.; BEN-YEHOSHUA, S. Effect of combined application of heat treatments and plastic packaging on keeping quality of 'Oroblanco' fruit (Citrus grandis L. x C. paradisi Macf.). Postharvest Biology and Technology, Wageningen, v.20, p.287-294, 2000.

RODOV, V.; BEN-YEHOSHUA, S.; ALBAGLI, R.; FANG, D.Q. Reducing chilling injury and decay of stored citrus fruit by hot water dips. Postharvest Biology and Technology, Wageningen, v.5, p.119-127, 1995.

SALA, J.M.; LAFUENTE, M.T. Catalase in the heat-induced chilling tolerance of cold-stored hybrid Fortune mandarin fruits. Journal of Agriculture and Food Chemistry, Easton, v.47, p.2410-2414, 1999.

SCHIRRA, M.; COHEN, E. Long-term storage of 'Olinda' oranges under chilling and intermittent warming temperatures. Postharvest Biology and Technology, Wageningen, v.16, p.63-69, 1999.

SCHIRRA, M.; MULAS, M. 'Fortune' mandarin quality following prestorage water dips and intermittent warming during cold storage. HortScience, Alexandria, v.30, n.3, p.560-551, 1995. 